تأثير اسانس درمانى استنشاقى گياه اسطوخودوس بر شدت افسردگى و ناتوانى ناشى از سردرد در بيماران مبتلا به ميخرن: يك كار آزمايى بالينى تصادفى سلى

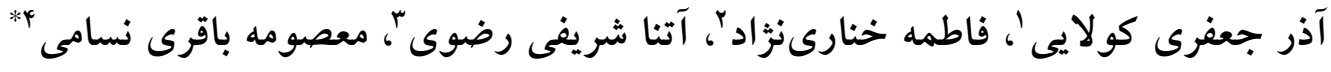

1- دانشجوى كارشناسى ارشد يُرستارى سالمندى، كميته تحقيقات دانشجويى، دانشكاه علوم يزشكى مازندران،

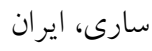

r- كارشناسى يرستارى، كميته تحقيقات دانشجويى، دانشخاه علوم يزشكى مازندران، سارى، ايران

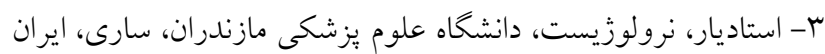

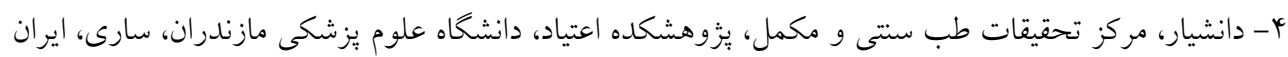

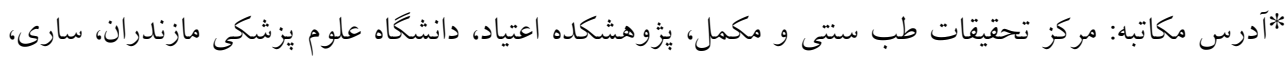

ايران

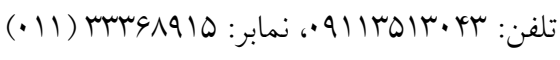
anna3043@gmail.com : بست الكترونيك :

doi: 10.29252/jmp.2.70.162 9V/4/M1

تاريخ دريافت: 9V/M/T9

مقدمه: ناتوانى ناشى از سردرد در ميخرن و مزمن شدن آن مى تواند منجر به ايجاد حالات غم و اندوه و افسردحى شوند.

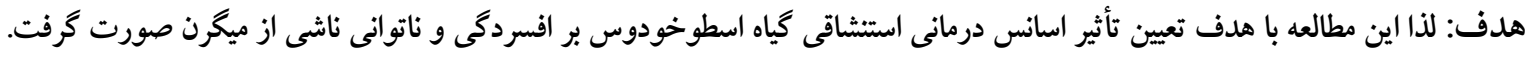

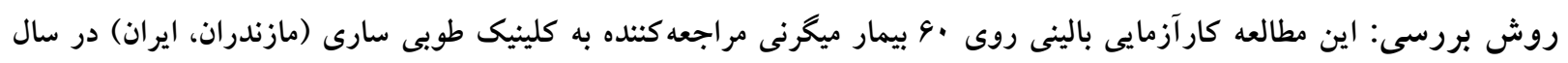

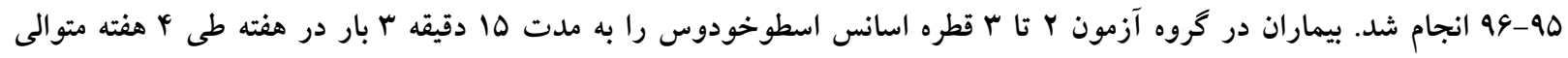

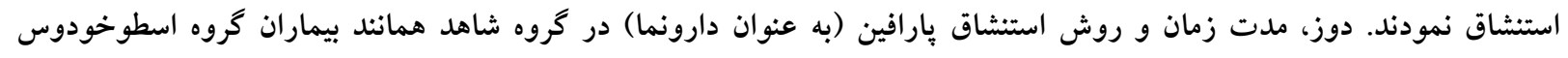

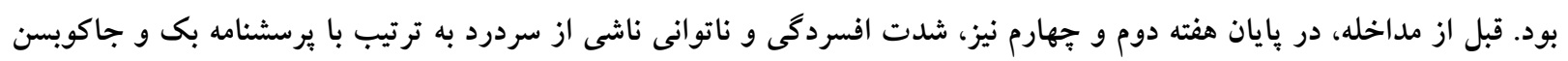

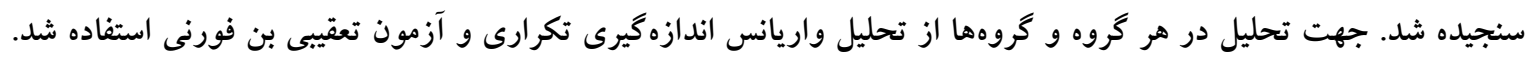

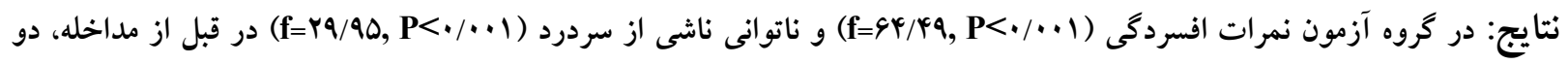

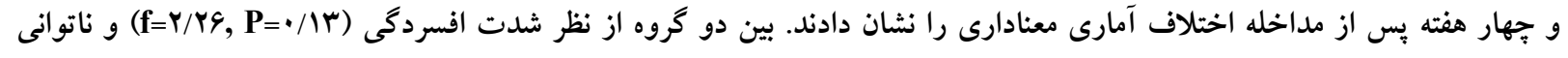

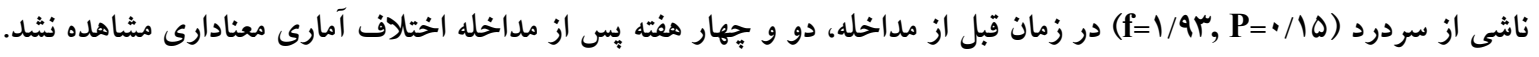

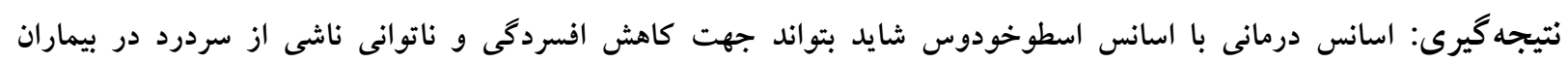
ميكرنى مفيد واقع شود.

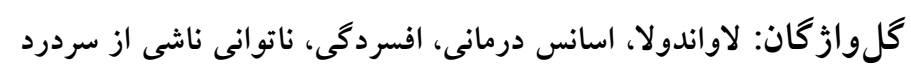




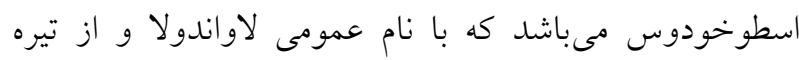

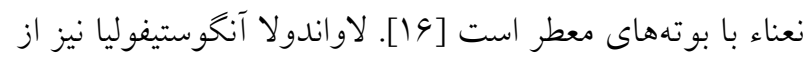

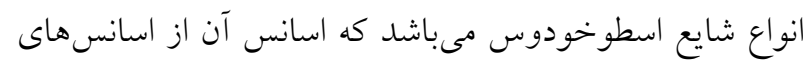

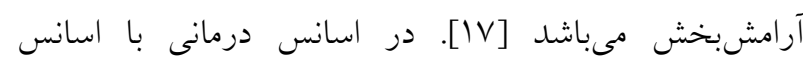

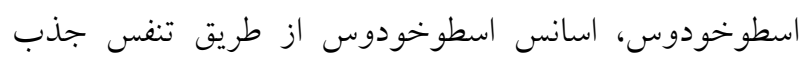

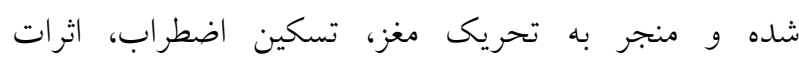

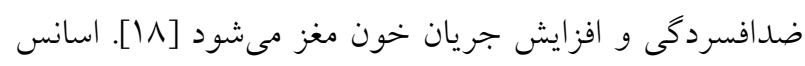

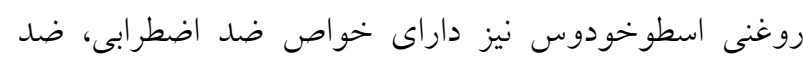

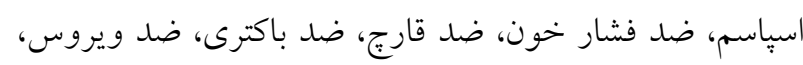

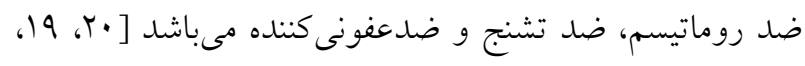

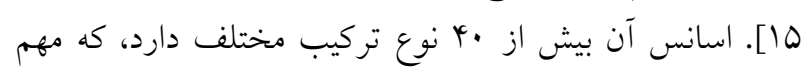

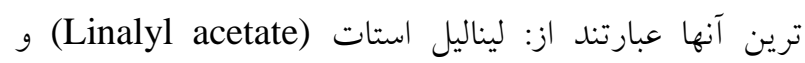

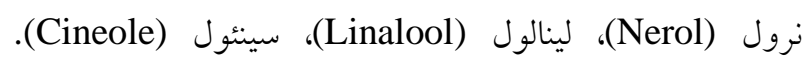

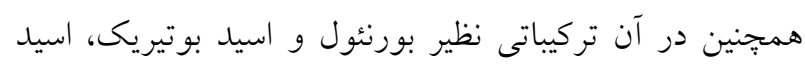

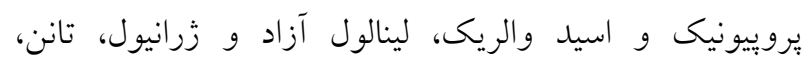

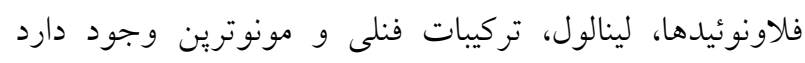

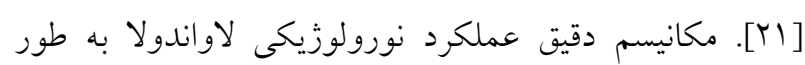
كامل مشخص نشده اما ممكن است اثراتى مشابه با بنزوديازيين

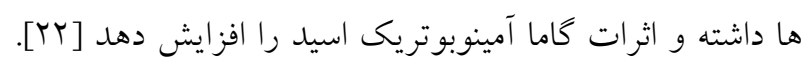

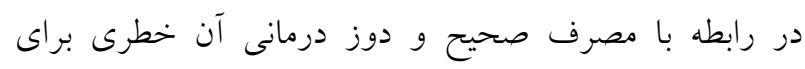

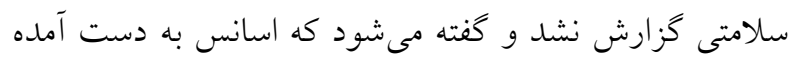

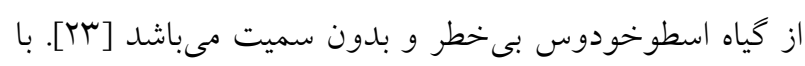

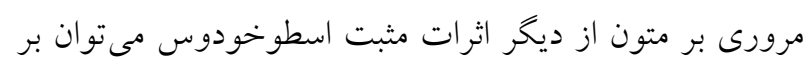
كاهش شدت سردردهاى ميكرنى [YYY] و افسردكى بيماران

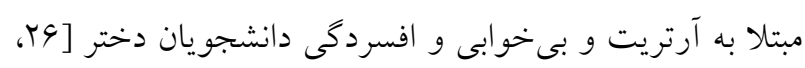

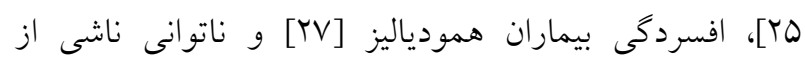

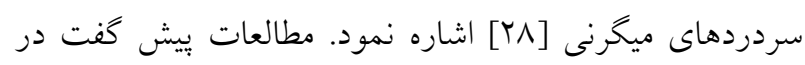

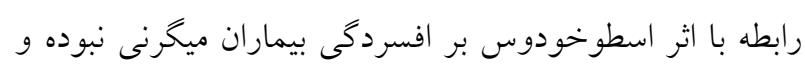

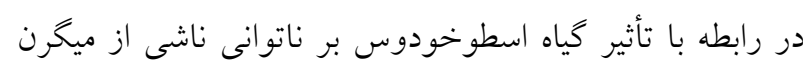

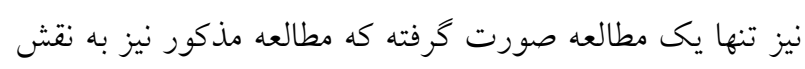

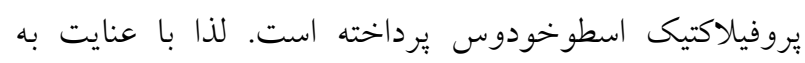

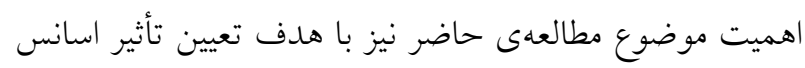

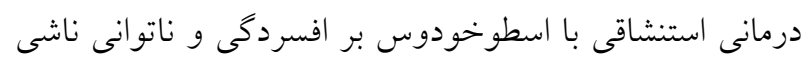

سالانه FY ميليون نفر در سراسر جهان از / أ ميليارد حمله

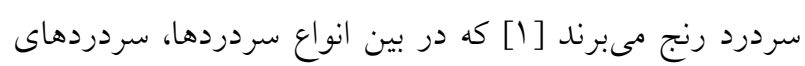

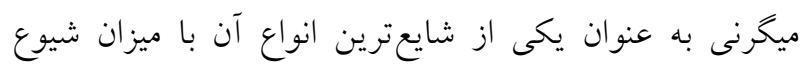

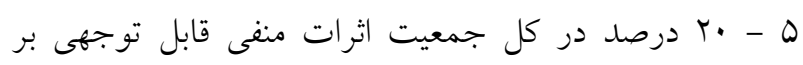

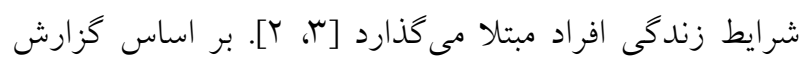

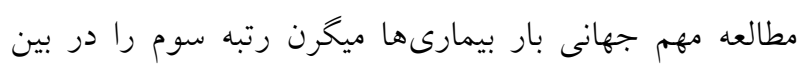

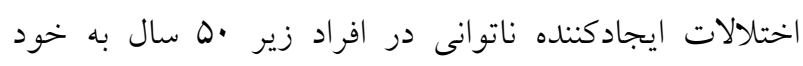

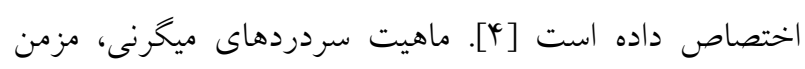

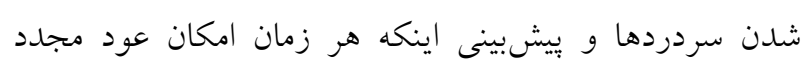

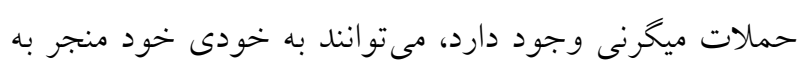

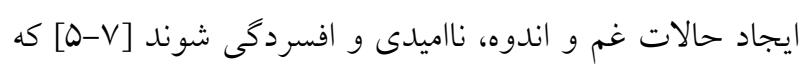

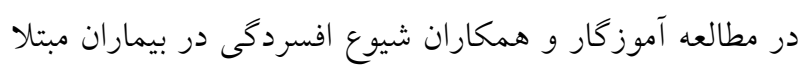

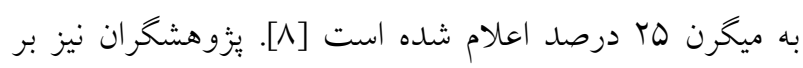

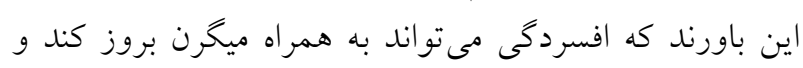

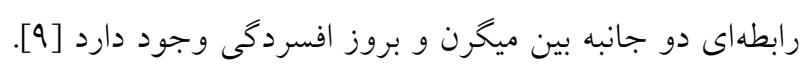

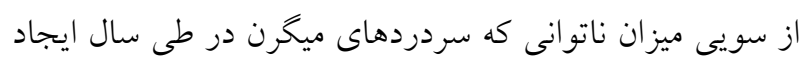

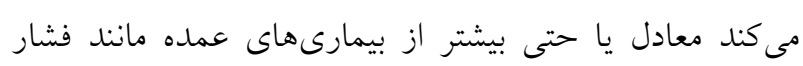

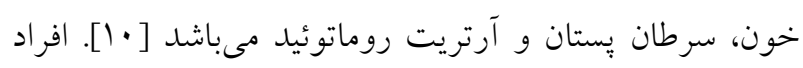

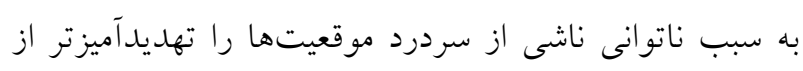

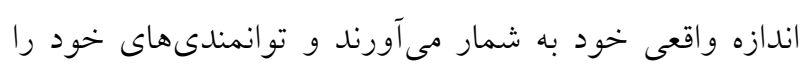

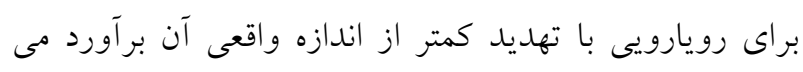

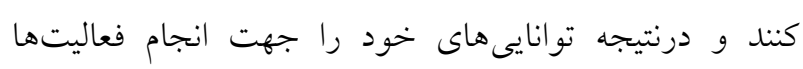
محدودتر از حد معمول تصور مىنمايند [11]

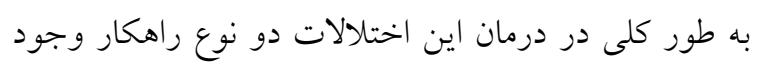

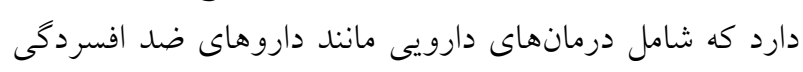

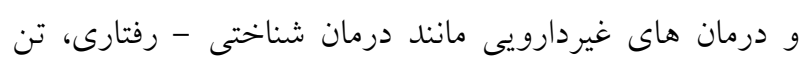

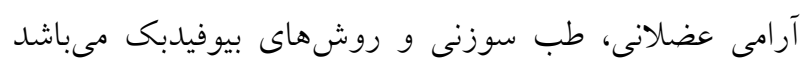

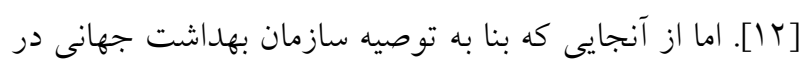

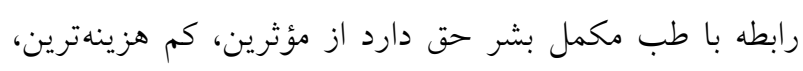

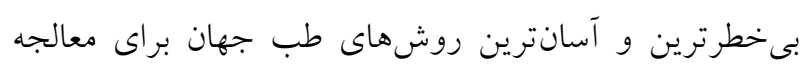

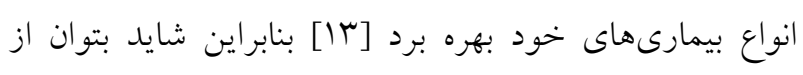

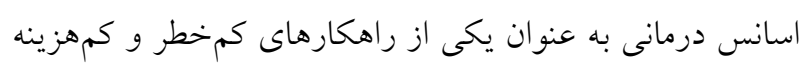

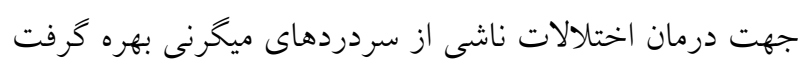


شد. هنخام نمونه گيرى از M نقر بيمار مبتلا به ميخرن مراجعه

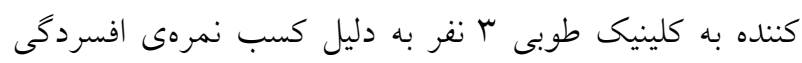

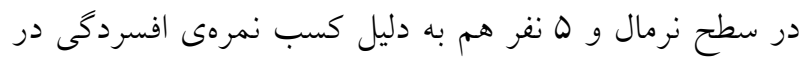

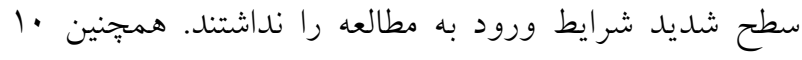

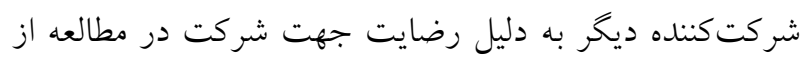

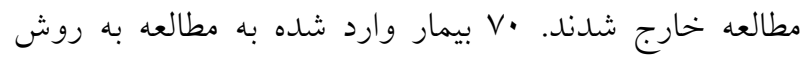

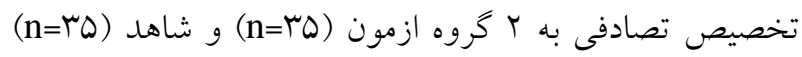

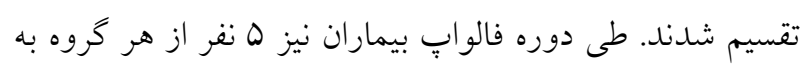

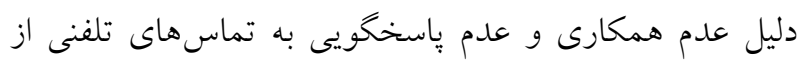

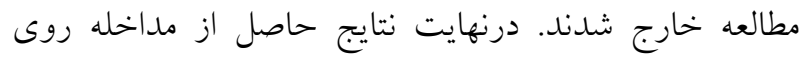
شصت بيمار مورد تجزيه و تحليل قرار گرفت (نمودار شماره

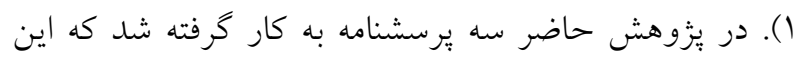

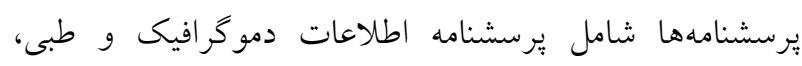

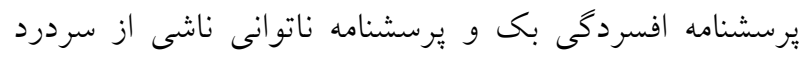

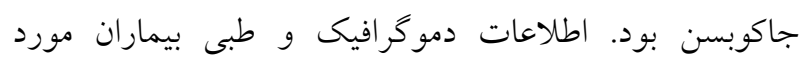

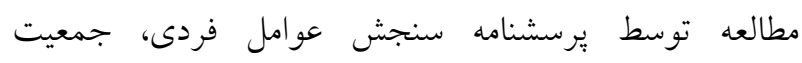

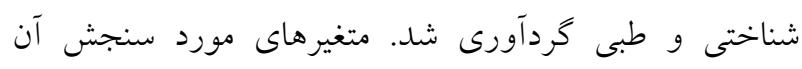

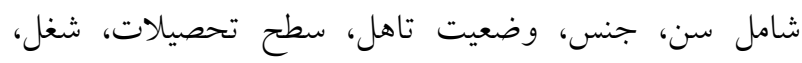

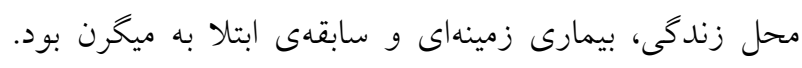

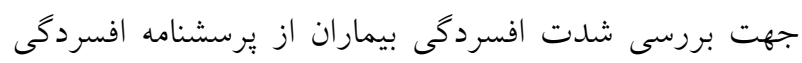

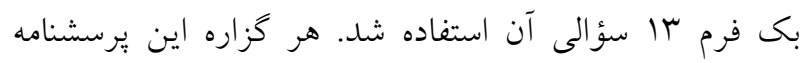

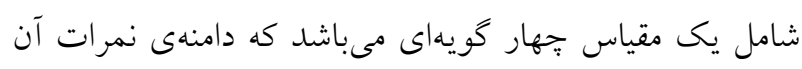

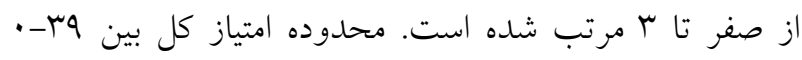

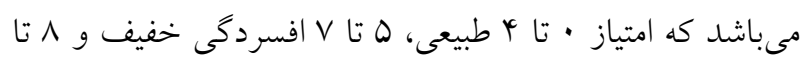

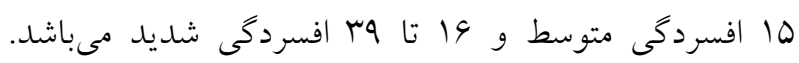

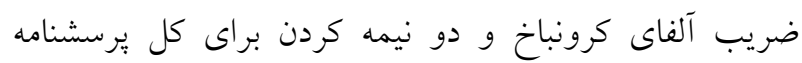

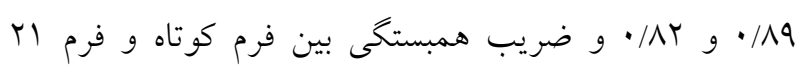

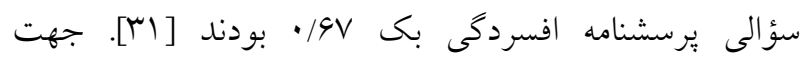

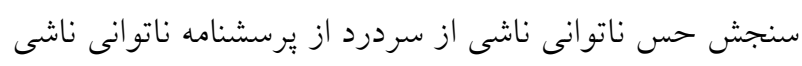

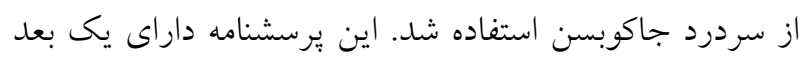

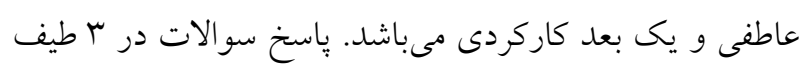

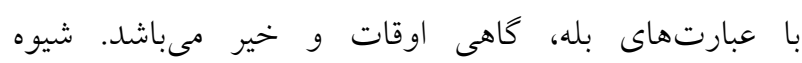

از ميخرن در بيماران ميخرنى صورت گرفت تا شايد بتوان كامى

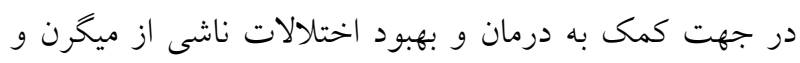
ارتقا كيفيت زندكى اين دسته از افراد در جامعه برداشت دون.

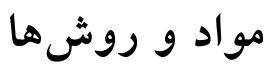

مطالعهى حاضر به صورت كارآزمايى بالينى تصادفى

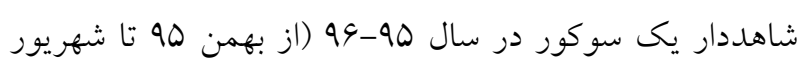

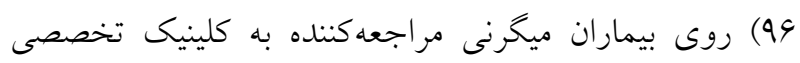

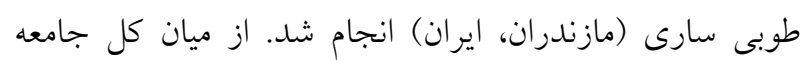

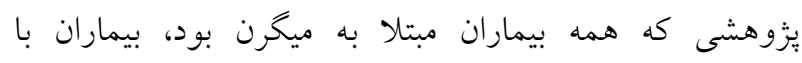

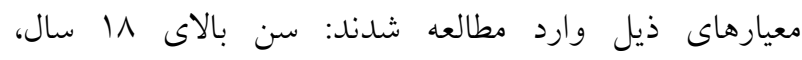

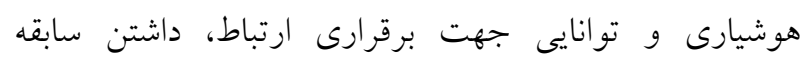

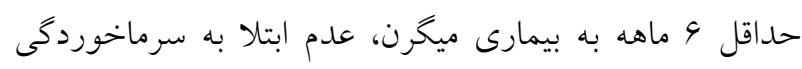

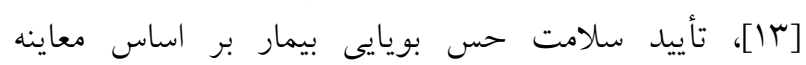

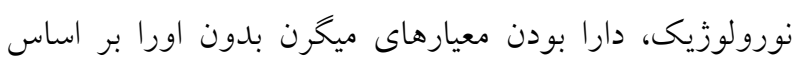

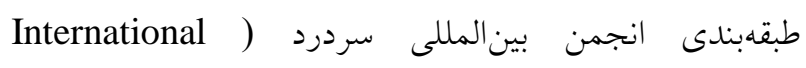
(Headache Society

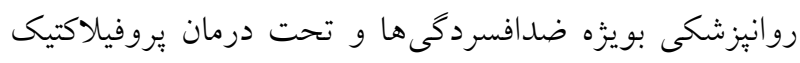

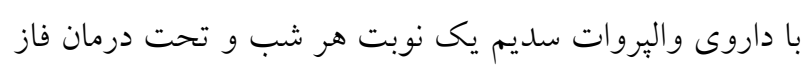

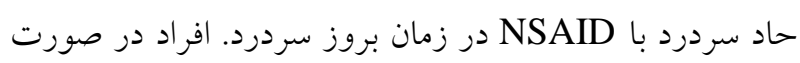

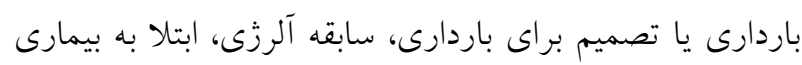

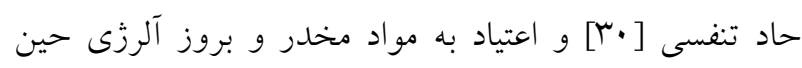

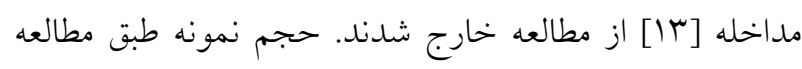

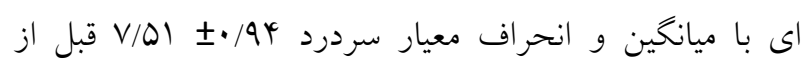

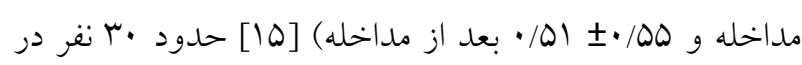

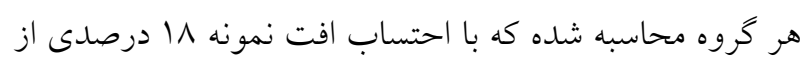

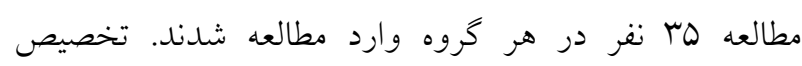

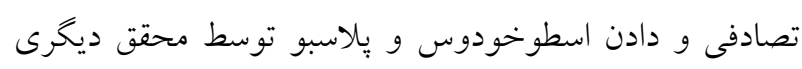

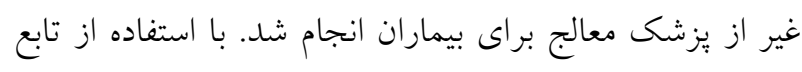

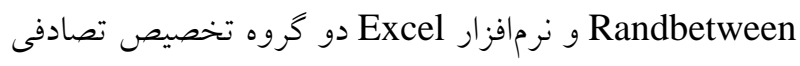

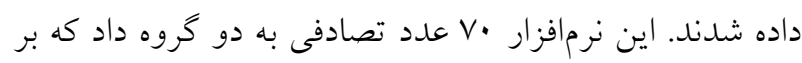

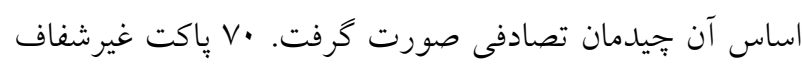

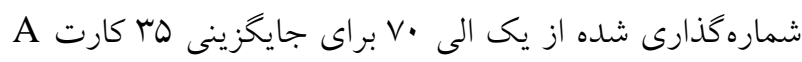

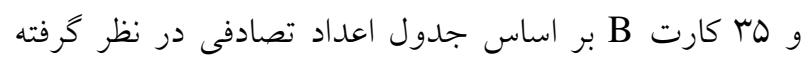


تأثير اسانس درمانى ...

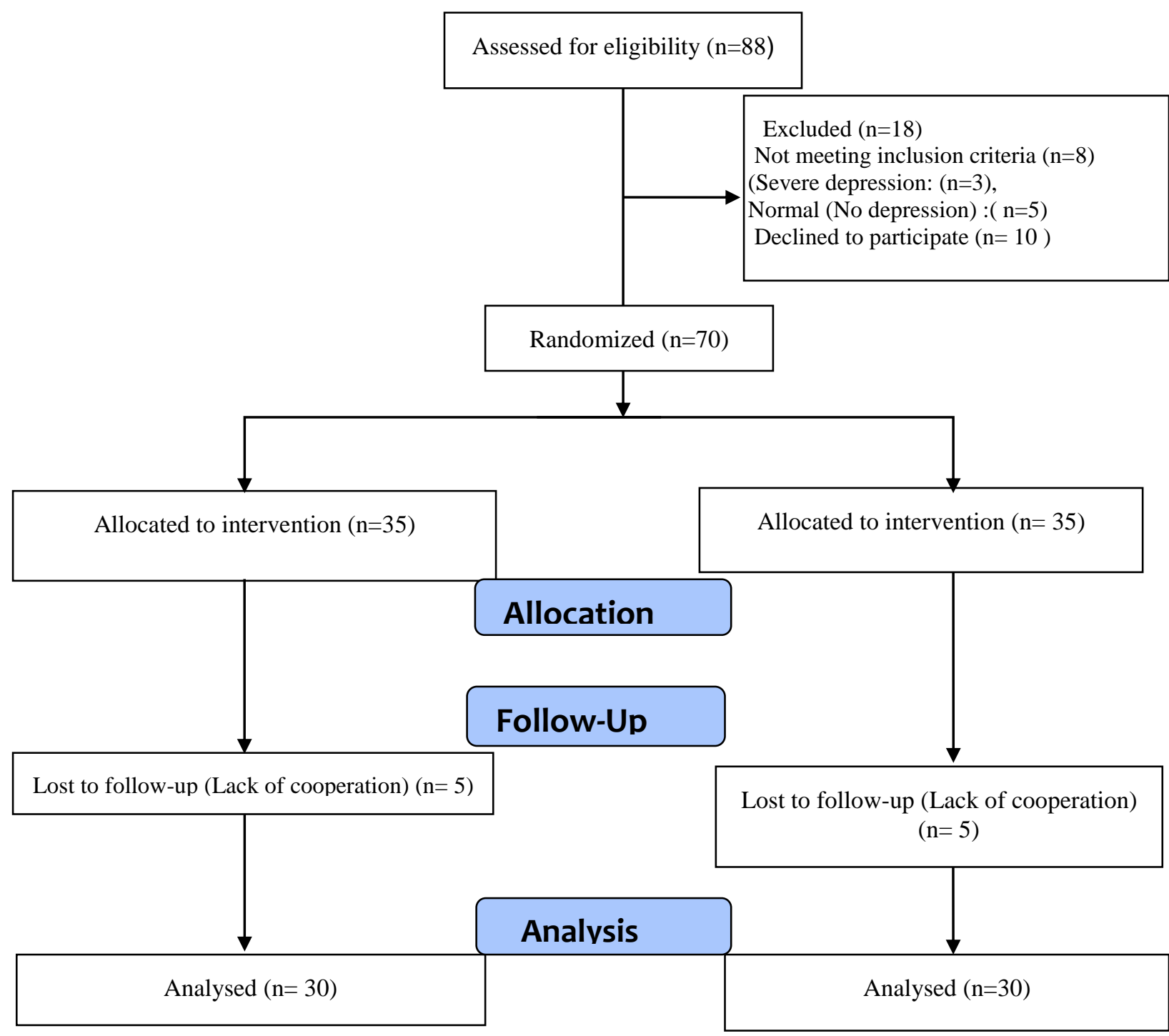

نمودار شماره ا- نحوهى تخصيص تصادفى نمونهها در دو گروه آزمون و شاهد (كنسورت)

مقياس Scl-25 (فرم بتا) نيز ضرايب همبستخى خرده مقياس

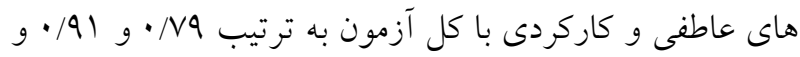

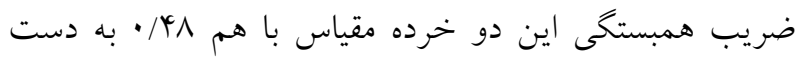

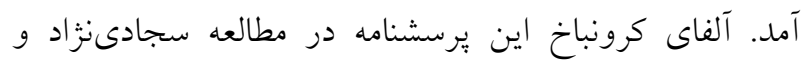

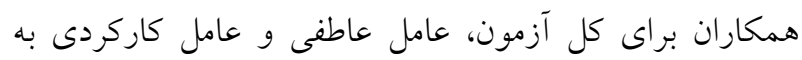

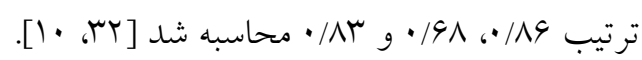

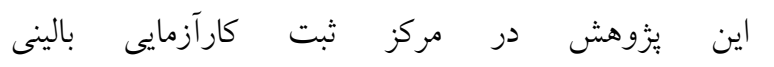
(Iranian Registry of Clinical Trials) (www.irct.ir) كد IRCTID: IRCT201608117494N22 به ثبت رسيده

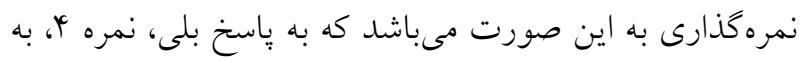

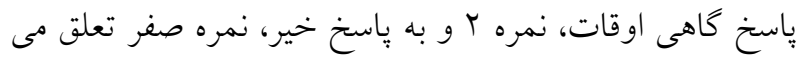

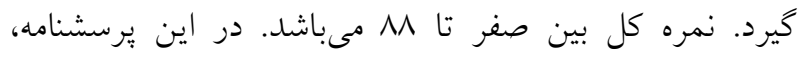

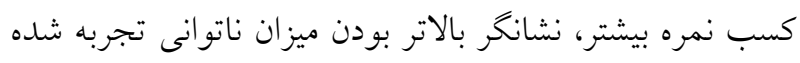

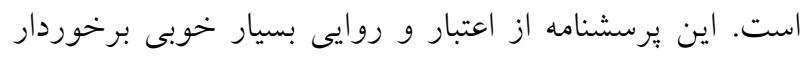

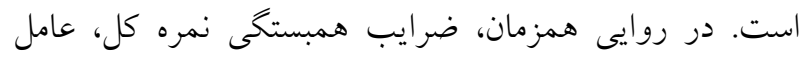

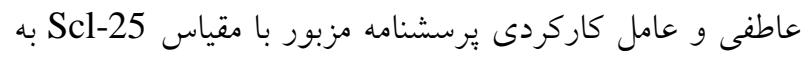

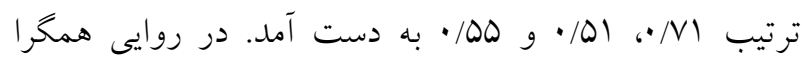

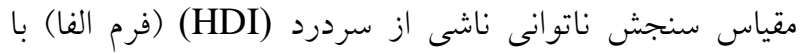


افسردگى در گروه آزمون در طى زمانهاى قبل، دو هفته و جهار

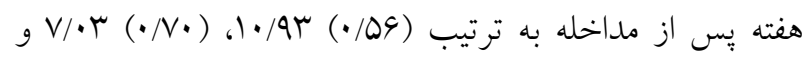

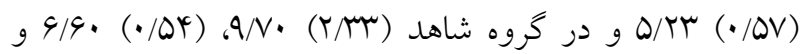

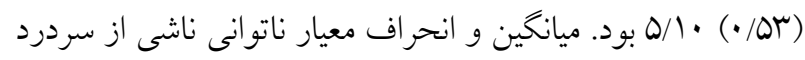

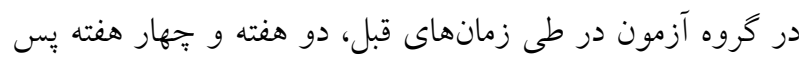
از مداخله به ترتيب (r/A9)

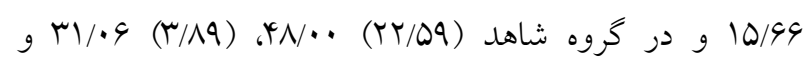
(r/9r)

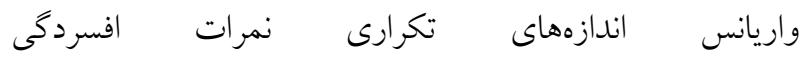

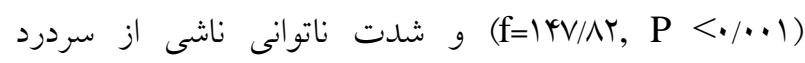

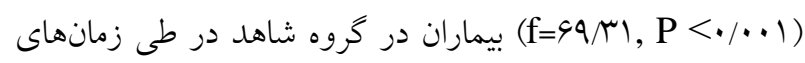
قبل، دو هفته و جهار هفته يس از مداخله اختلاف آمارى معنادارى

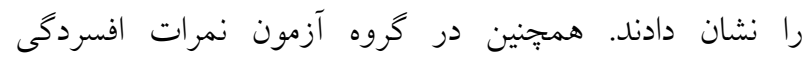

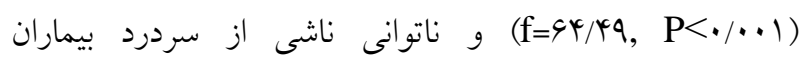
(f=rq/90, P<•/・1)

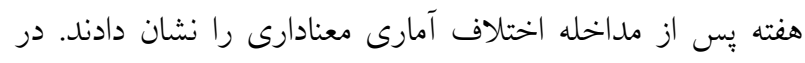

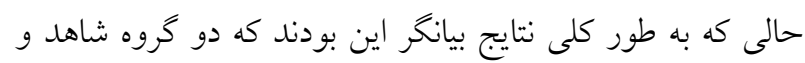

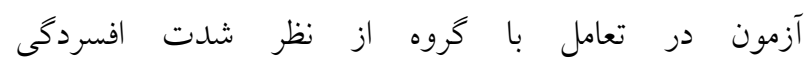

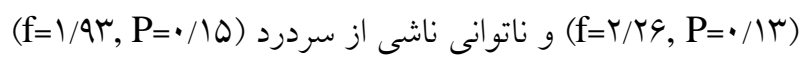

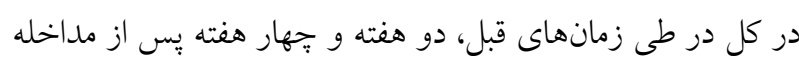
اختلاف آمارى معنادارى نداثتند.

طبق نتايج حاصل از آزمون بن فورنى در گروه آزمون شدارت

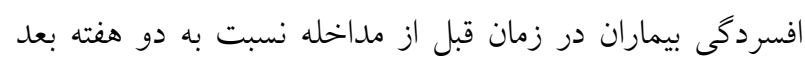

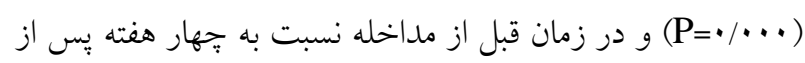

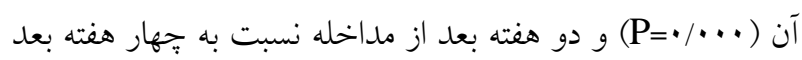

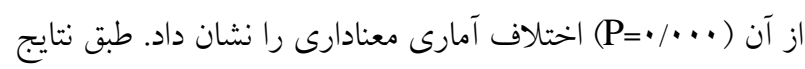

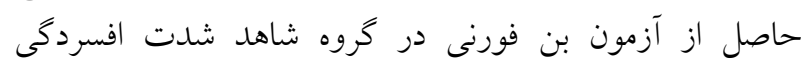

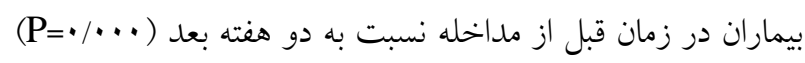

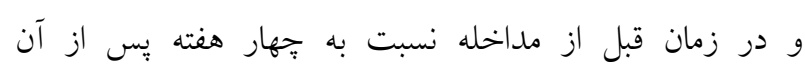

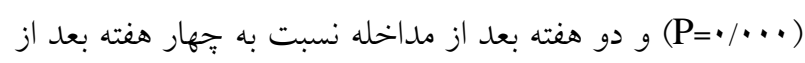

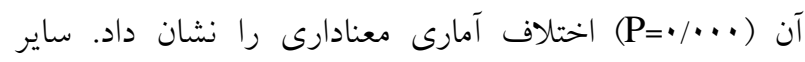

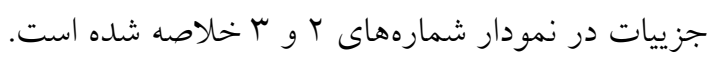

است. يّ از ارائه اطلاعات در مورد نحوه انجام مطالعه و اهداف

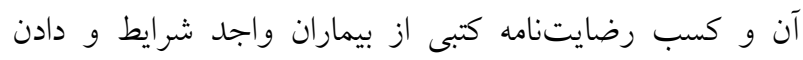
اطمينان به آنها از محرمانه بودن اطلاعات، نمونه كيرى انجام اندام شد.

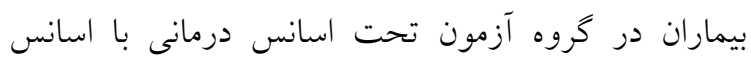
روغنى اسطوخودوس تهيه شده از شركت داروسازى باريج اسانس كه داراى كواهينامه كيفيت 9001 ISO و و استاندارد ISO/IEC 17025 در ب روز هفته به صورت يك در ميان در روزهاى شنبه،

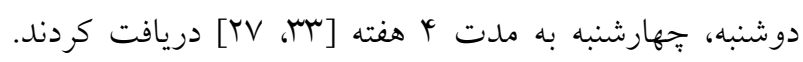

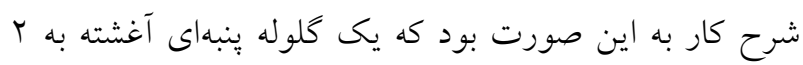

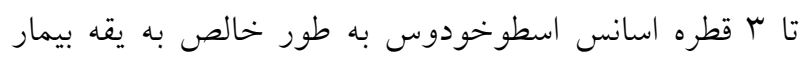

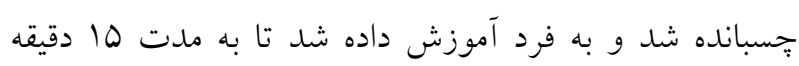

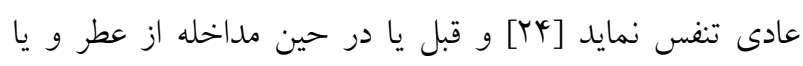

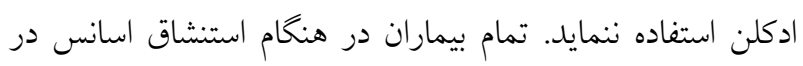

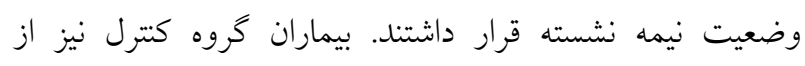

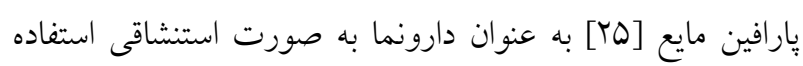

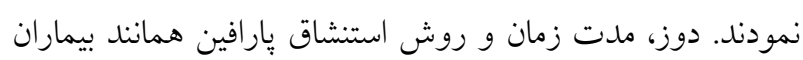

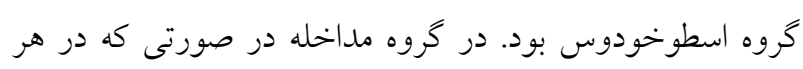

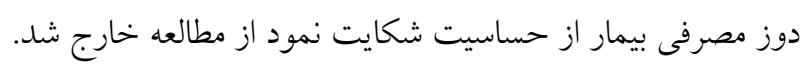

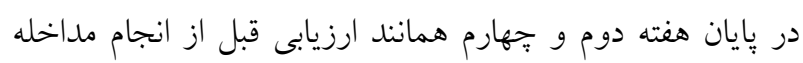

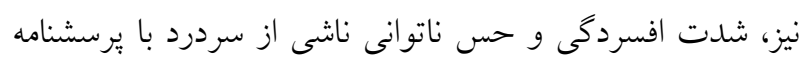

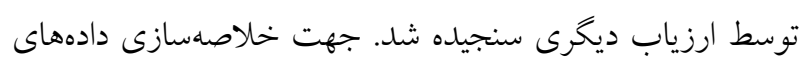

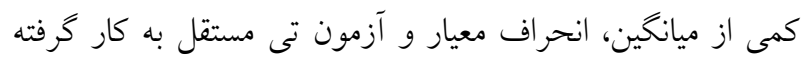

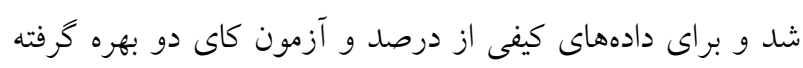

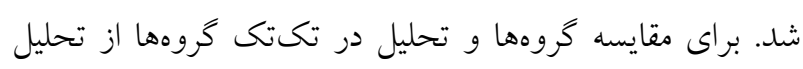
واريانس اندازهيرى تكرارى و بن فورنى استفاده شد.

\section{نتايج}

نتايج حاصل از مطالعه حاضر نشان دادند كه دو گروه آزمون

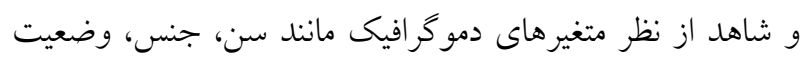

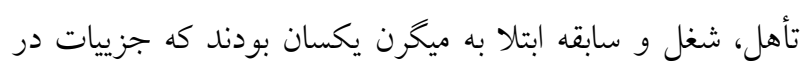
جدول شماره ا خلاصه شده است. ميانخين و انحراف معيار 
تأثير اسانس درمانى ...

جدول شماره ا - اطلاعات جمعيت شناختى و طبى بيماران

\begin{tabular}{|c|c|c|c|}
\hline$P$ value & كروه كنترل & كروه آزمون & 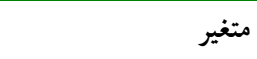 \\
\hline $\mathrm{P}=\cdot / 9 \pi$ & $r \Delta / N T(\mid r / 99)$ & $r V / l \cdot(9 / r I)$ & سن \\
\hline \multirow{3}{*}{$\mathrm{P}=\cdot / 9$} & & & 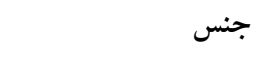 \\
\hline & $r(\% \cdot \cdot)$ & $1(/ \mu / \mu)$ & مرد \\
\hline & $T V(\% \cdot \cdot)$ & rq $(/ .99 / \mathrm{V})$ & زن \\
\hline \multirow{3}{*}{$\mathrm{P}=\cdot / \Delta$} & & & وضعيت تاهل \\
\hline & $0(\% 19 / \mathrm{N})$ & $r(\% .1 \Gamma / \mu)$ & مجرد \\
\hline & TQ $(\% / \wedge \Gamma / \Gamma)$ & $r q(/ / \Lambda \& / V)$ & متاهل \\
\hline \multirow{3}{*}{$\mathrm{P}=\cdot / \Delta$} & & & 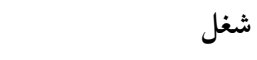 \\
\hline & $\wedge(/$ Y Y $/ \mathrm{V})$ & $V(\%, \mu / \Gamma)$ & شاغل \\
\hline & $r(\%(/ N r / r)$ & $r(\%(/ . V 9 / V)$ & بيكار \\
\hline $\mathrm{P}=\cdot / T \Lambda$ & $1 / 4 \cdot(\cdot / 49)$ & $1 / r \&\left(\cdot / K^{4}\right)$ & سابقدى ابتلا به ميخرن \\
\hline
\end{tabular}

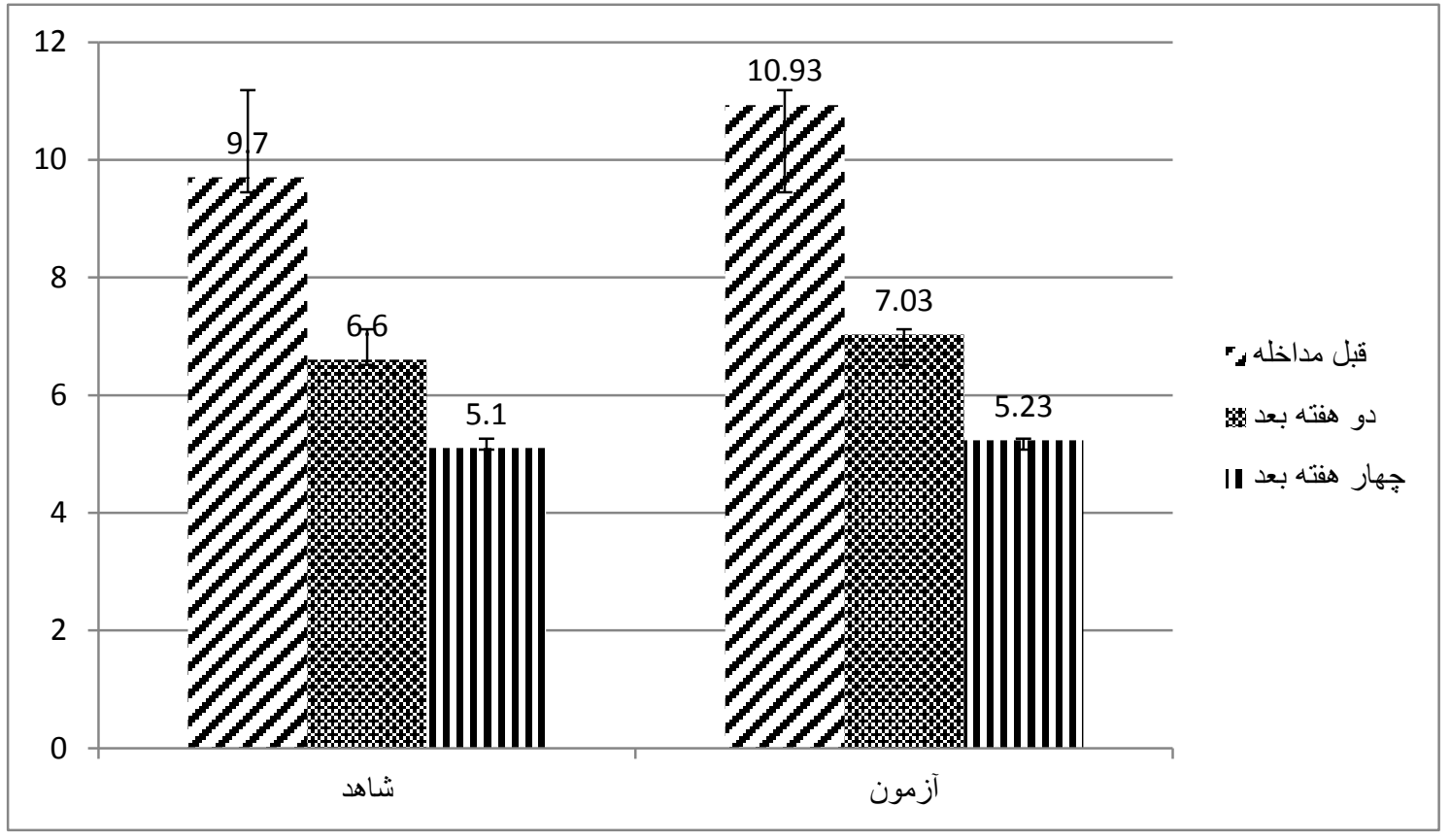

نمودار شماره ץ- مقايسهى نمرات افسردگى دو گروه آزمون و شاهد در طى زمانهاى قبل از مداخله، دو هفته و جهار هفته بعد از مداخله 


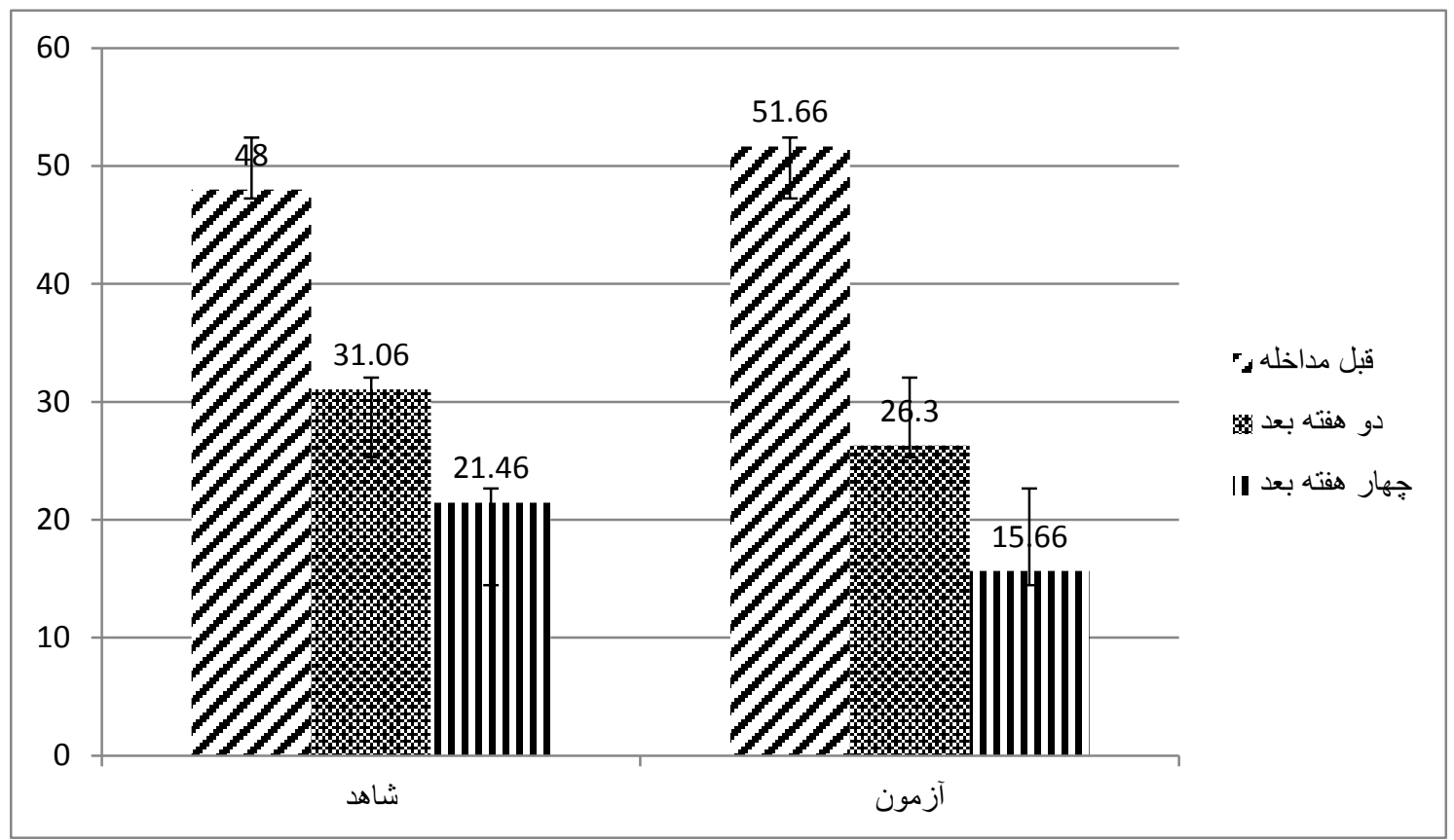

نمودار شماره بـ- مقايسهى نمرات ناتوانى ناشى از سردرد دو گروه آزمون و شاهد در طى زمانهاى قبل از مداخله و دو هفته و جهار هفته بعد از مداخله

اظهار نمودند كه اسانس درمانى استنشاقى با اسانس روغنى

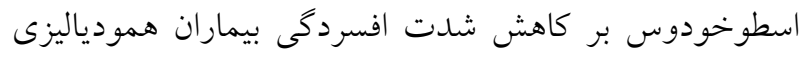

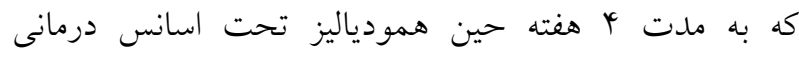

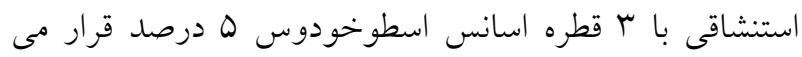

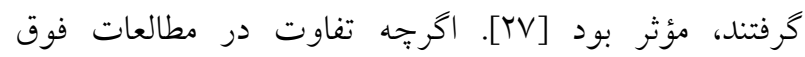

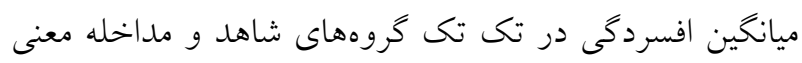
دار شد ولى متفاوت با نتايج مطالعه حاضر مقايسه ميانخين افسردگى در دو گروه معنى دار نشد. از علل احتمالى تفاوت در نتايج مطالعات فوق با مطالعه حاضر شايد بتوان به جامعاده يُزوهش و ابزار سنجش متغيرهاى موردنظر اشاره نمود. ابزار

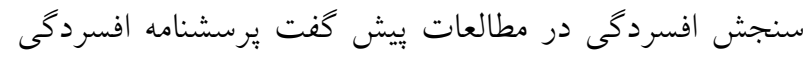

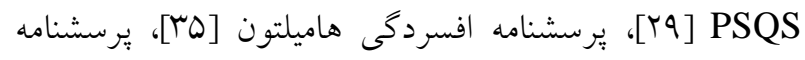

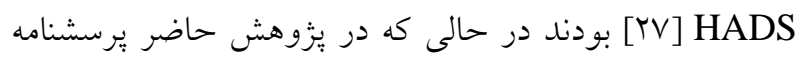

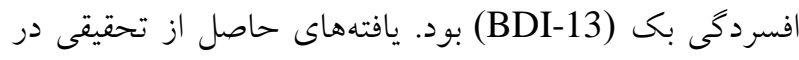

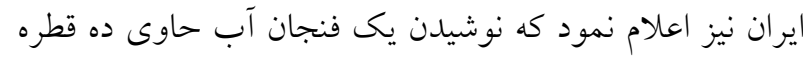
اسانس اسطوخودوس هر شب طى سه ماه توانست ميزان

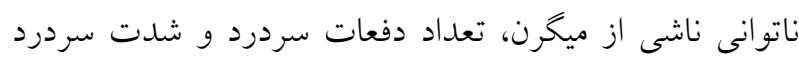

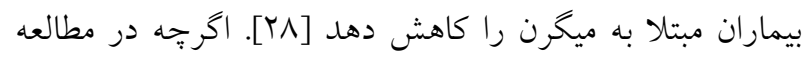

نتايج مطالعه حاضر حاكى از عدم وجود تغييرات معنىدار

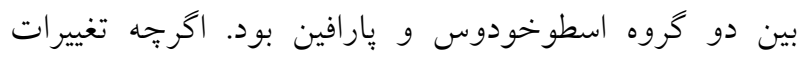
معنى دار در نمرات افسردگى و ناتوانى ناشى از سردرد در دو و برون جهار هفته يس از مداخله نسبت به زمان قبل از مداخله در

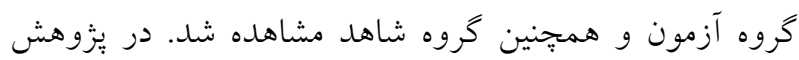

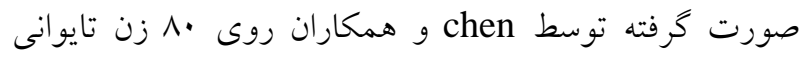

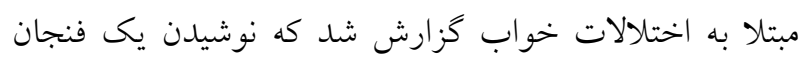

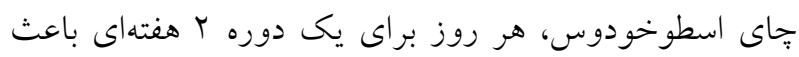

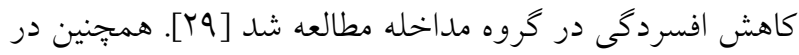

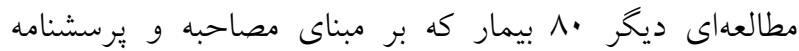

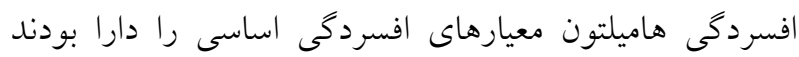

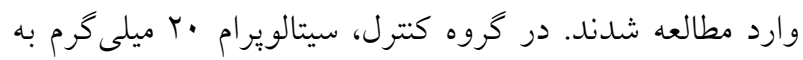

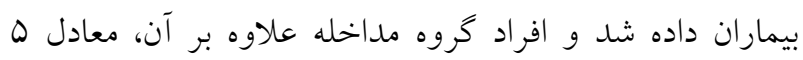

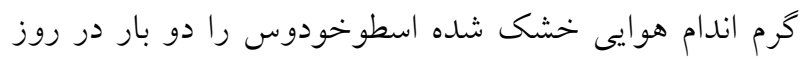

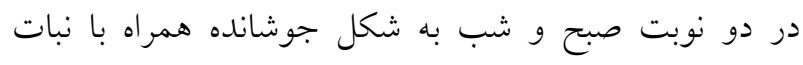
دريافت كردند. بعد از انجام مداخله ميانكين افسردكى كاهش

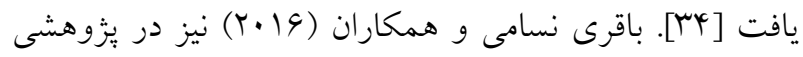


بيماران و همراهى آنان طى يكى دورهى جهار هفتهاى از طريق

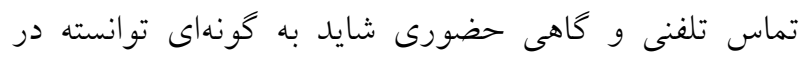

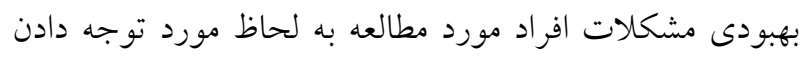
و حمايت بعد روانى افراد شركت كننده نقش داشته باشد. لذا

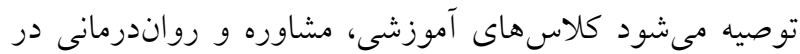
كنار درمان دارويى اين بيماران مورد توجه قرار كيرد.

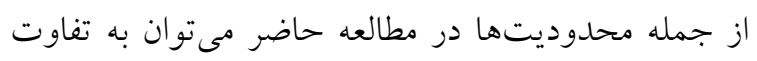

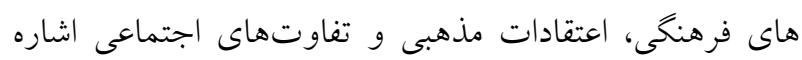

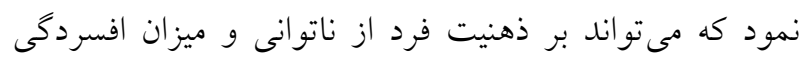
بيماران مؤثر باشد، ولى سعى شد از طريق تخصيص تصادفى دهى

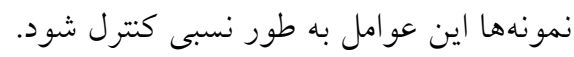
مطالعه حاضر نشان داد كه اسانس درمانى با اسانس روغنى بر بانى

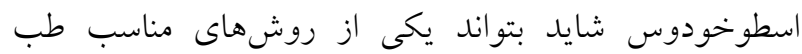

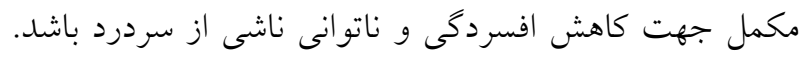

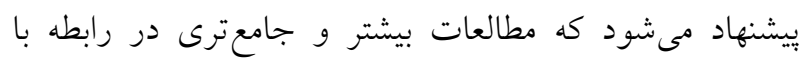

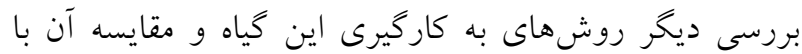
ساير روشهاى روان درمانى صورت كيرد.

\section{تشكر و قدردانى}

از بيماران و تمامى كسانى كه ما را در انجام مطالعهى

حاضر يارى نمودند، صميمانه قدردانى مىشود. هم:خنين نويسندكان مراتب تقدير و تشكر خود را از واحد توسعه تحقيقات بالينى بيمارستان بوعلى سينا و معاونت تحقيقات

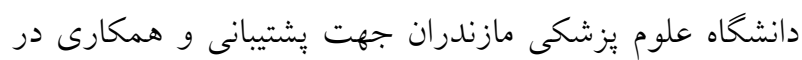

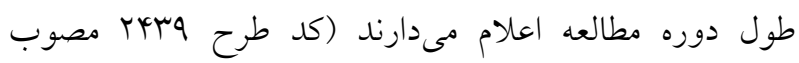
(IRCTID: IRCT201608117494N22). (9ه/N/9

1. Akbarzadeh D, Rajabzadeh A, Amini Beyrami $\mathrm{H}$ and $\mathrm{A}$ AM. Comparing the mean score of depression, sleep quality and resiliency in patients with migraine and tension type headaches with
فوق تفاوت در ميانخين ناتوانى ناشى از ميخرن در تك تكى

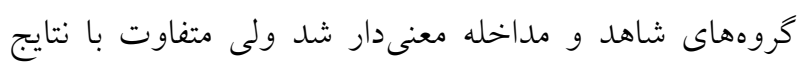

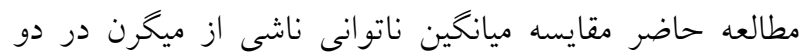

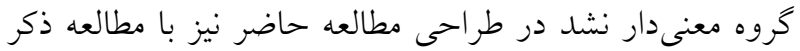
شده تفاوتهايى وجود دارد كه مى توان به طول مدت مداخله و

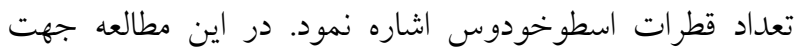

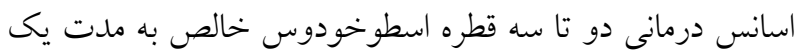

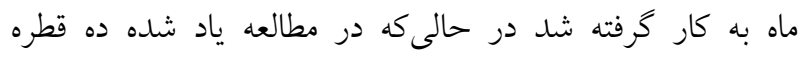

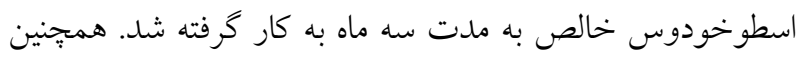
جهت سنجش ناتوانى ناشى از سردرد در مطالعه فوق از يرسشنامه MIDAS ناتوانى ناشى از سردرد جاكوبسن استفاده شد.

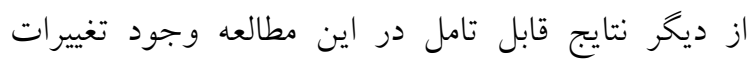

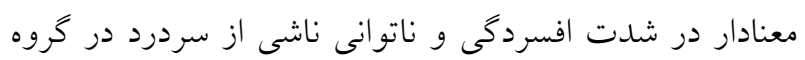

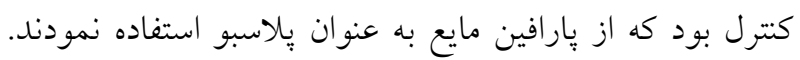

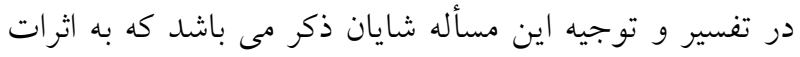
تلقينى پِلاسبو بر بيماران مورد مطالعه اشاره نماييم. يافتههاى

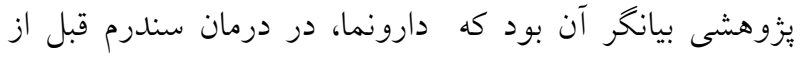

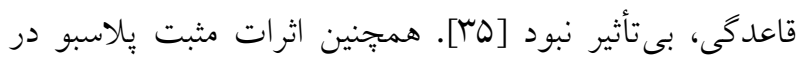

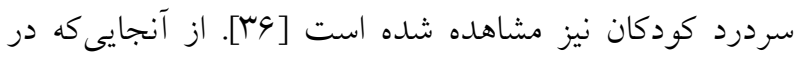

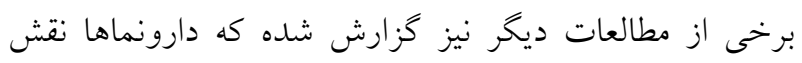

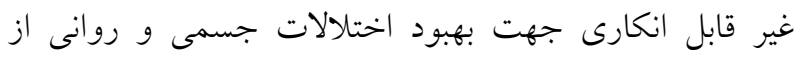

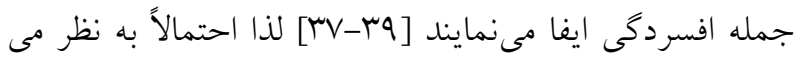
رسد كه در اين مطالعه نيز از اثرات پِاسبو در جهت بهان بهبود افسردحى و ناتوانى ناشى از سردرد بيماران بهره خرفته شد. از از

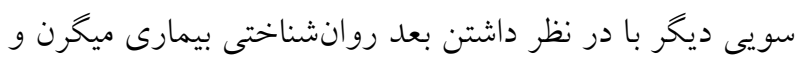

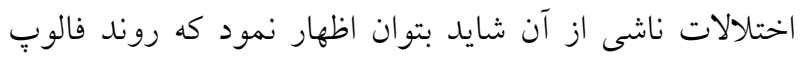

\section{منابع}

healthy individuals. Anesthesiology and Pain Medicine 2015; 4 (41): 41-32.

2. Ahmadpanah $\mathrm{M}$, Kakekhani $\mathrm{H}$ and Ghaderzadeh P. Investigation of prevalence and clinical characteristics of migraine patients referred 
to the neurology department of Sina hospital. Pajouhan Scientific J. 2010; 12 (4): 1-6.

3. Güngen BD, Aras YG, Gül SS, Türkan Acar, Ayaz AB, Alagöz AN and et al. The effect of maternal migraine headache on their children's quality of life. Acta Neurologica Belgica 2017; 117 (3): 687-94.

4. Ashina S, Serrano D, Lipton RB, Maizels M, Manack AN, Turkel CC and et al. Depression and risk of transformation of episodic to chronic migraine. J. Headache Pain. 2012; 13 (8): 615-24.

5. Almalki ZA, Alzhrani M AG, Altowairqi AT, Aljawi YA, Fallatah SA, Assaedi LM and et al. Prevalence of Migraine Headache in Taif City, Saudi Arabia. J. Clinical Medicine Res. 2018; 10 (2): 125-33.

6. Eraslan D, Dikmen PY, Aydınlar EL and İncesu

C. The relation of sexual function to migrainerelated disability, depression and anxiety in patients with migraine. The Journal of Headache and Pain 2014; 15: 32.

7. Sadeghniiat Kh, Rajabzadeh A, Ghajarzadeh M and Ghafarpour M. Sleep quality and depression among patients with migraine. Acta Medica Iranica 2013; 51 (11): 784-8.

8. Amoozegar F, Patten SB, Becker WJ, Bulloch AGM, Fiest KM, Davenport WJ and et al. The prevalence of depression and the accuracy of depression screening tools in migraine patients. General Hospital Psychiatry 2017; 48: 25-31.

9. Breslau N, Lipton RB, Stewart WF, Schultz LR and Welch K. Comorbidity of migraine and depression: investigating potential etiology and prognosis. Neurol. 2003; 60 (8): 1308-12.

10. Saedi $S$ and Abolghasemi A. Effect of guided visualization and progressive relaxation training on pain and disability in women with migraine. Journal of Woman and Culture 2011; 3 (10): 39-52.

11. Sajadinejad M , Mohammadi N, Taghavi SMR and Ashgazadeh N. Therapeutic Effects of Cognitive-Behavioral Group Therapy on headache disability and depression in patients with migraine and tension headache. Iranian Journal of Psychiatry and Clinical Psychol. 2009; 14 (4): 411-8.

12. Danaie Sij Z, Dehghani Firoozabadi $M$ and Sharifzadeh G. Effects of cognitive-behavioral stress management on depression, anxiety, and pain control in patients with migraine headaches. Modern Care J. 2013; 10 (3): 157-64.

13. Zarifnejad GhH, Eshghi E, Mirhaghi AH and Ghorbanzadeh $\mathrm{H}$. The effect of aromatherapy with Lavender essential oil in treatment of headache due to Nitroglycerine infusion in patient admitted to cardiac emergency department. Complementary Medicine J. 2015; 3 (16): 1250-4.

14. Ghods AA, Sotodehasl N, Ghorbani R and Valian M. The Effects of Aroma on the Accuracy of Intensive Care Nurses. J. Babol Univ. Med. Sci. 2013; 15 (5): 45-8.

15. Zarifnejad Gh, Eshghi E, Mirhaghi AH and Ghorbanzadeh $\mathrm{H}$. The effect of aromatherapy with Lavender essential oil in treatment of headache due to Nitroglycerine infusion in patient admitted to cardiac emergency department. Complementary Medicine J. 2015; 3 (16): 1250-4.

16. Heidari Gorji M, Ashrastaghi OM, Habibi V, Yazdani J and Ebrahimzadeh M. The effectiveness of lavender essense on strenotomy related pain intensity after coronary artery bypass grafting. $A d v$. Biomed. Res. 2015; 4: 127.

17. Najafi Z, Taghadosi M, Sharifi KH, Farrokhian $A$ and Tagharrobi $Z$. The effects of Inhalation Aromatherapy on Anxiety in Patients with Myocardial Infarction: A Randomized Clinical Trial. Iran. Red. Crescent. Med. J. 2014; 16 (8): 1-3.

18. Vakilian K, Karamat A, Mousavi A, Shariati M, Ajami M and Atarha M. The effect of Lavender essence via inhalation method on labor pain. $J$. Shahrekord Univ. Med. Sci. 2012; 14 (1): 34-40.

19. Hasanzadeh F, Kashouk NM, Amini S, Asili J, Emami SA, Vashani HB and et al. The effect of cold application and lavender oil inhalation in 
تأثير اسانس درمانى ...

cardiac surgery patients undergoing chest tube removal. EXCLI J. 2016; 15 (64): 64-74.

20. Kerachian N, Alaee H, Gharavi-Naini M, Pilevarian A and Moghimi A. Effects of alcoholic extract of Avena sativa, Hypericum perforatum, Passiflora incarnata and Lavandula officinalis on symptoms of morphine withdrawal syndrome in rats. Physiology and Pharmacol. 2007; 10 (4): 313-21.

21. Hadipour A, Hoseini Mazinani $M$ and Mehrafarin A. Changes in Essential Oil Content/Composition and Shoot Aerial Yield of Lavender (Lavandula officinalis L.) Affected by Different Treatments of Nitrogen. J. Med. Plants 2013; 2 (46): 156-69.

22. Mohammad Khani Shahri L, Sabet Birjandi S, Shahri HM. Effect of massage Aromatherapy with lavandula on the duration of first and second stage of labor in nulliparous women. HMJ. 2013; 17 (2): 145-54.

23. Cetinkaya $B$ and Basbakkal Z. The effectiveness of aromatherapy massage using lavender oil as a treatment for infantile colic. Int. J. Nurs. Pract. 2012; 18 (2): 164-9.

24. Sasannejad P, Saeedi M, Shoeibi A, Gorji A, Abbasi $M$ and Foroughipour $M$. Lavender Essential Oil in the treatment of migraine headache: A Placebo-Controlled Clinical Trial. European Neurol. 2012; 67: 288-91.

25. Kim MJ, Nam ES and Paik S. The effects of aromatherapy on pain, depression, and life satisfaction of arthritis patients. Taehan Kanho Hakhoe Chi 2005; 35 (1): 186-94.

26. Lee IS and Lee G. Effects of lavender aromatherapy on insomnia and depression in women college students. Journal of Korean Academy of Nursing 2006; 36 (1): 136-43.

27. Bagheri-Nesami M, Shorofi SA, Nikkhah A and Espahbodi $\mathrm{F}$. The effects of lavender essential oil aromatherapy on anxiety and depression in haemodialysis patients. Pharm. Biomed. Res. 2017; 3 (1): 8-13.
28. Rafie SH, Namjoyan F, Golfakhrabadi F, Yousefbeyk F and Hassanzadeh A. Effect of lavender essential oil as a prophylactic therapy for migraine: a randomized controlled clinical trial. Journal of Herbal Medicine. 2016; 6 (1): 18-23.

29. Chen SL and Chen C. Effects of Lavender Tea on Fatigue, Depression, and Maternal-Infant Attachment in Sleep-Disturbed Postnatal Women. Worldviews Evid. Based Nurs. 2015; 12 (6): 370-9. 30. Bahraini S, Naji A, Mannani R and Bekhradi R. The comparison of the effects of effleurage massage with aromatic oil and non-aromatic oil on fatigue severity in women with multiple sclerosis. J. Urmia Nurs. Midwifery Fac. 2011; 9 (5): 327-36.

31. Rajabi GR. Psychometric Properties of Beck Depression Inventory Short Form Items (BDI-13). J. Iranian Psychologists 2005; 1 (4): 30-8.

32. Sajadinejad M, Mohammadi N and Ashgahzadeh N. The evaluation of psychometric properties of headache disability inventory in the headache patients. J. Shahrekord Univ. Med. Sci. 2007; 9 (1): 55-62.

33. Bagheri-Nesami M, Shorofi SA, Nikkhah A, Espahbodi $\mathrm{F}$ and Koolaee FG. The effects of aromatherapy with lavender essential oil on fatigue levels in haemodialysis patients: A randomized clinical trial. Complementary Therapies in Clinical Practice 2016; 22: 33-7.

34. Nikfarjam P and Osarzadgan N. The effect of lavender (Lavandula angustifolia Mill) in the treatment of depression. Iran. Red Crescent Med J. 2010; 11 (4): 66-73.

35. Pakgohar M, Mehran A, Salehi Sour Moghi M and Akhondzadeh S, Ahmadi M. Comparison of hypericum perforatum and placebo in treatment of physical symptoms of premenstrual syndrome. Hayat 2004; 10 (3): 31-7.

36. El-Chammas K, Keyes J, Thompson N, Vijayakumar J, Becher $\mathrm{D}$ and Jackson $\mathrm{J}$. Pharmacologic treatment of pediatric headaches: a meta-analysis. JAMA Pediatr. 2013; 167: 250-8. 
37. Colagiuria B, Schenkb LA, Kesslerc MD, Migraine Clinic. The Journal of Pediatrics 2014; Dorseydef SG and Colloca L. The placebo effect: 165 (4): $659-65$.

From concepts to genes. Neuroscience 2015; 307:

39. Miller FG, Colloca L and Kaptchuk T. The 171-90.

placebo effect: illness and interpersonal healing.

38. Faria V, Linnman C, Lebel A and Borsook D.

Perspect Biol. Med. 2009; 52 (4): 518.

Harnessing the Placebo effect in Pediatric 
تأثير اسانس درمانى ...

\title{
The Effect of Aromatherapy with Lavender Essence on Depression and Headache Disability in Migraine Patients: A Randomized Clinical Trial
}

\author{
Jafari-Koulaee A (M.Sc. Student) ${ }^{1}$, Khenarinezhad F (B.Sc. ${ }^{2}$, Sharifi Razavi A (M.D.) ${ }^{3}$, \\ Bagheri-Nesami M (Ph.D.) $)^{4^{*}}$
}

\begin{abstract}
1- MSc student in Geriatric Nursing, Research Committee, Mazandaran University of Medical Sciences, Sari, Iran

2- BSc in Nursing, Research Committee, Mazandaran University of Medical Sciences, Sari, Iran

3- Assistant Professor, Neurologist, Mazandaran University of Medical Sciences, Sari, Iran

4- Associate Professor, Traditional and Complementary Medicine Research Center, Addiction Institute, Mazandaran University of Medical Sciences, Sari, Iran

*Corresponding author: Traditional and Complementary Medicine Research Center, Addiction Institute, Mazandaran University of Medical Sciences, Sari, Iran

Tel: +98-911-3513043

E-mail: anna3043@gmail.com
\end{abstract}

\begin{abstract}
Background: Disability due to headache in migraine and being chronic of it can lead to sadness and depression.

Objective: This study was aimed to determine the effect of lavender aromatherapy on depression and headache disability.

Methods: This clinical trial study was conducted on 60 migraine patients referred to Toba Clinic of Sari (Mazandaran, Iran) during 2016-2017. Patients in the experimental group inhaled 2-3 drops of lavender essence for 15 min, three times a week for 4 consecutive weeks. The dose, duration and inhalation method of paraffin (as placebo) in control group were similar to those in the experimental group. Before the intervention, at the end of the second and fourth weeks, the intensity of depression and headache disability were measured by Beck Depression Inventory (BDI-13) and Jacobson questionnaire. For analyzing in each group and groups, repeated measure tests and Boneforeni were used.

Results: In experimental group, depression $(P<0.001, F=64.49)$ and headache disability scores ( $P<0.001, F=29.95)$ were significantly different in the pre intervention, two and four weeks after the intervention. No statistically significant differences were observed between the two groups in terms of the depression $(P=0.13, f=2.6)$ and headache disability $(P=0.15, f=1.93)$ scores before, two and four weeks after intervention.

Conclusion: Lavender essential oil can be useful for reducing depression and headache disability in migraine patients.
\end{abstract}

Keywords: Aromatherapy, Depression, Headache disability, Lavandula angustifolia

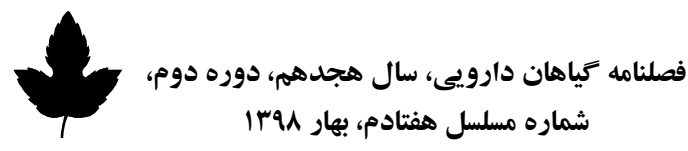

